Comm. in Asteroseismology

Vol. 152, 2008

\title{
BRITE-Constellation: Observation Planning
}

\author{
A. Kaiser ${ }^{1}$, R. Kuschnig ${ }^{1},{ }^{2}$
}

${ }^{1}$ Institut für Astronomie, Türkenschanzstrasse 17, 1180 Vienna, Austria

${ }^{2}$ Univ. of British Columbia, 6224 Agricultural Road, Vancouver V6T 1Z1, Canada

\section{Abstract}

This proceeding paper was generated using a Power-Point presentation from the workshop.

\section{Presentation Slides}

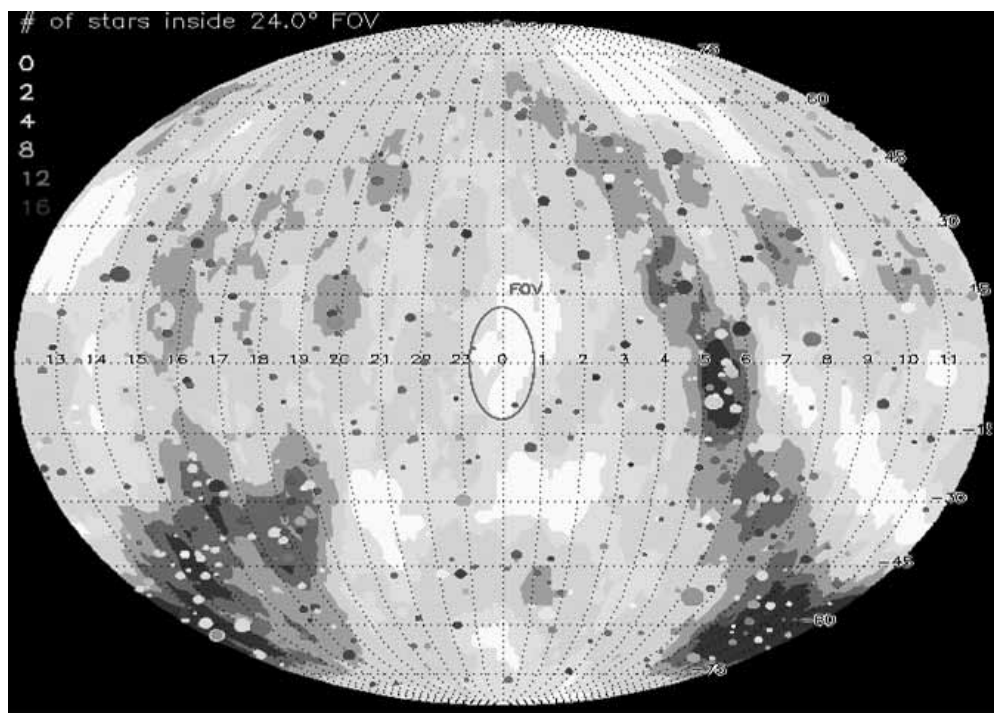

Figure 1: Plot of the whole sky for all 534 stars with a visual apparent magnitude brighter than $4^{m}$. Coded in levels of grey is the number of stars inside a field of view of $24^{\circ}$. 


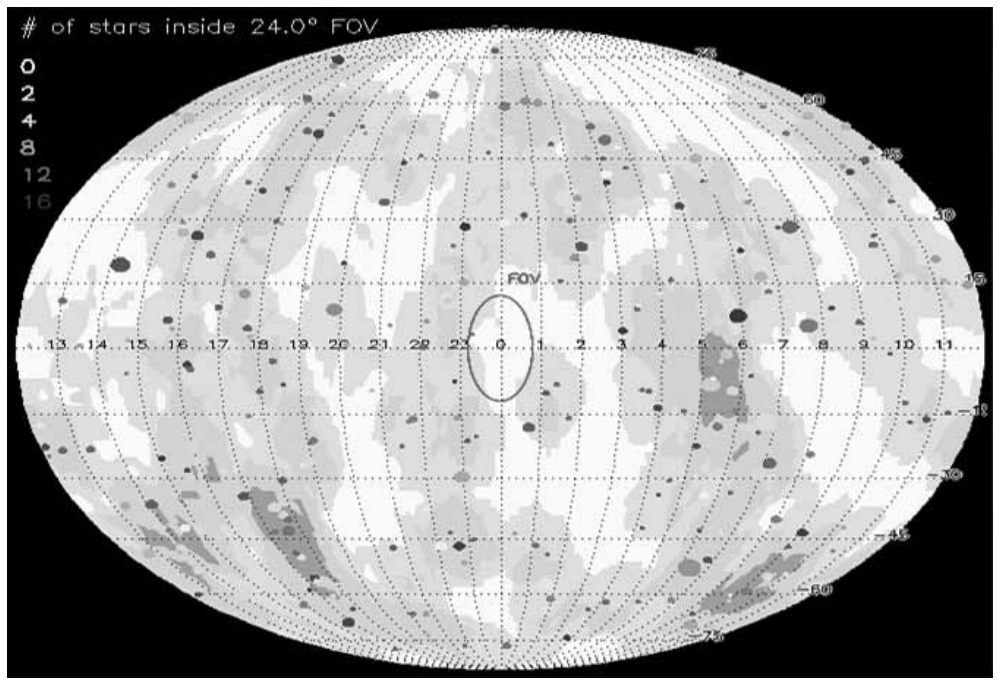

Figure 2: Same as Figure 1 but only for the 274 stars with less than $0.1 \%$ flux from polluting background objects.

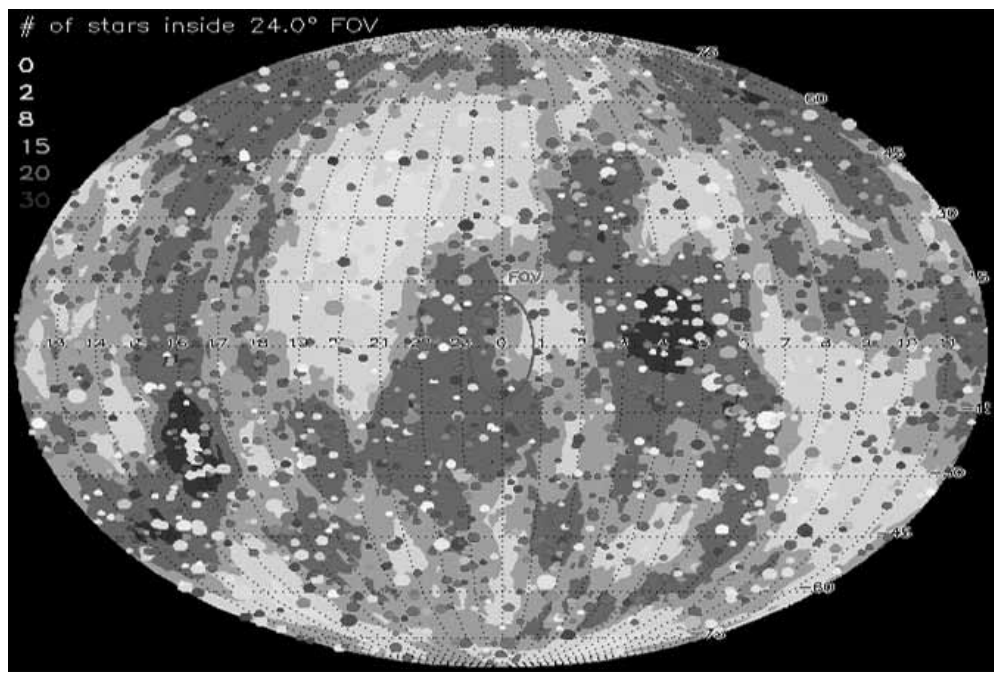

Figure 3: Same as Figure 2 but for stars with with a visual apparent magnitude brighter than $6^{m}$. 


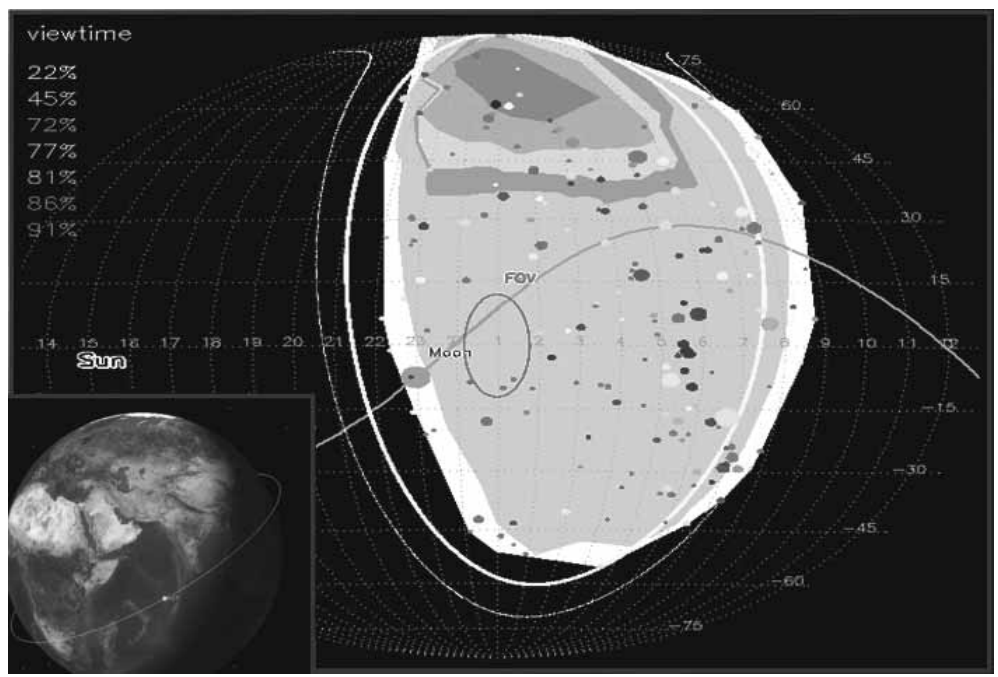

Figure 4: Unobstructed sky coverage for the Hubble Space Telescope for October 2008 coded in levels of grey.

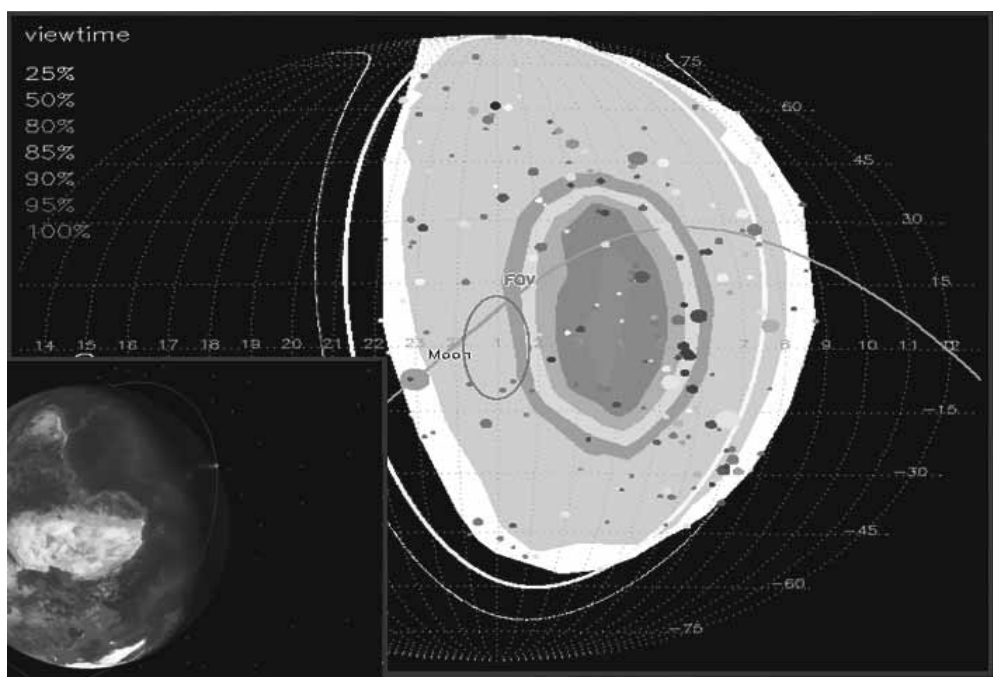

Figure 5: Same as Figure 4 but for the MOST Space Telescope. 


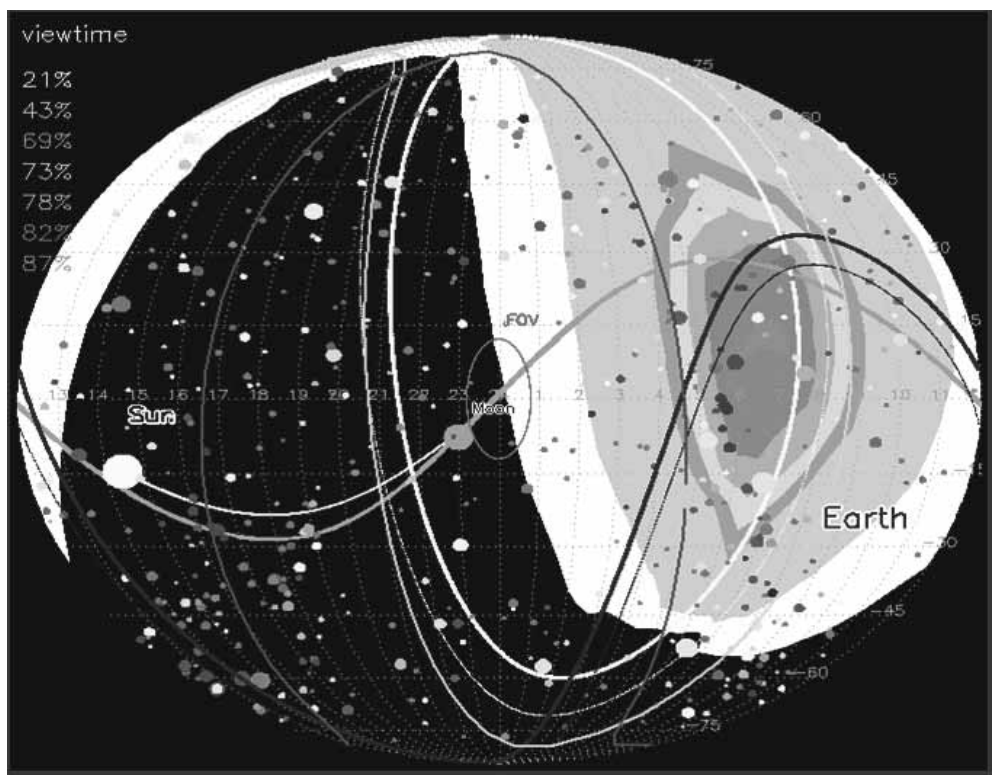

Figure 6: Same as Figure 5 but for 4 month.

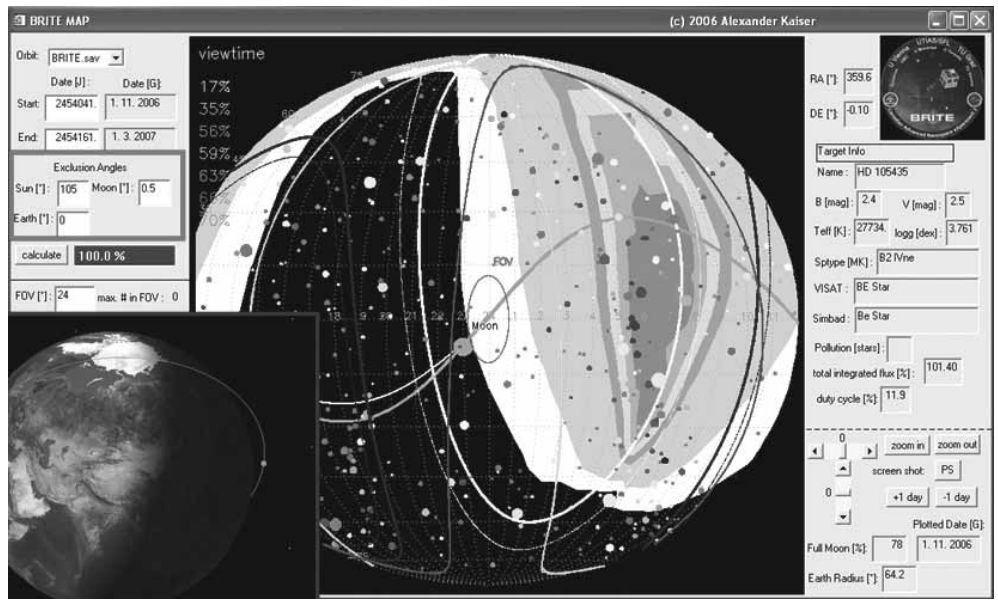

Figure 7: Same as Figure 6 but for a noon-midnight orbit. 


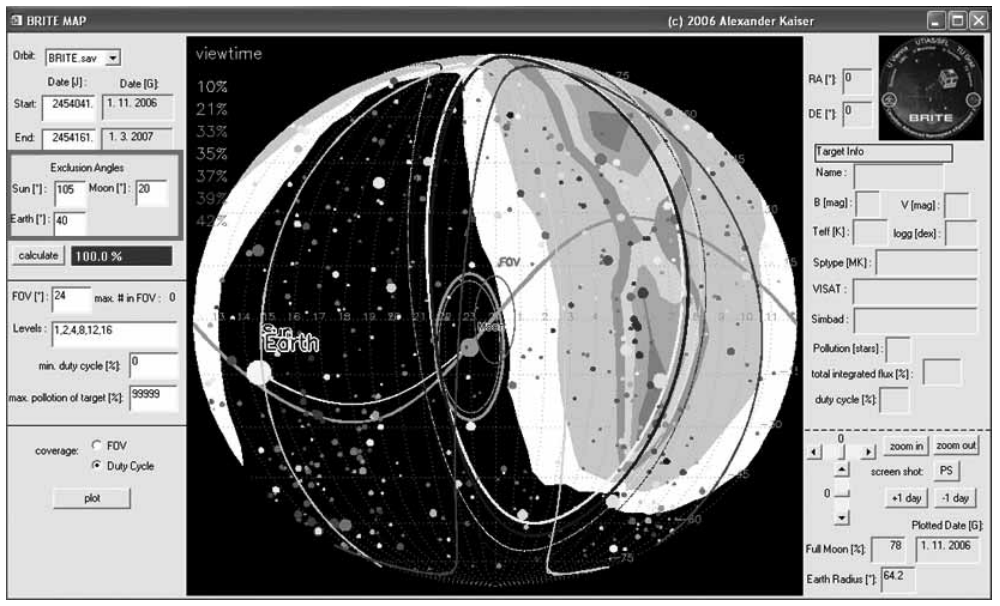

Figure 8: Same as Figure 7 but for larger earth and moon exclusion angles.

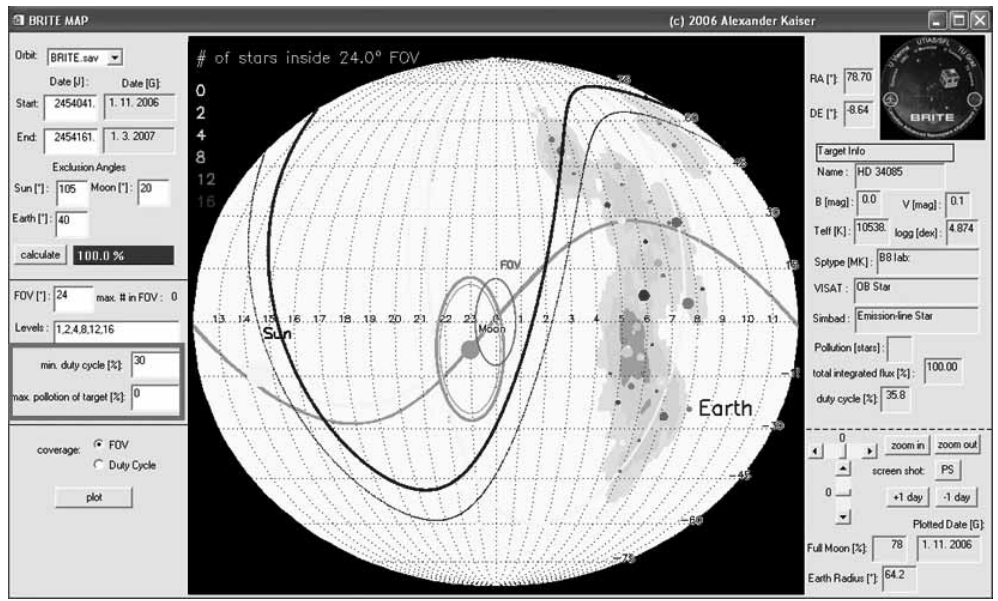

Figure 9: Plot of the whole sky coverage coded in levels of grey for targets with more than $30 \%$ viewtime and less than $0.1 \%$ flux from polluting background objects. 